\title{
Orientation behaviour of interchanges forming chiasmata in interstitial regions: A cytological approach
}

\author{
P. Arana, \\ N. Henriques-Gil* and \\ J. L. Santos
}

\author{
Departamento de Genética, Facultad de Biología, \\ Universidad Complutense de Madrid, 28040 Madrid, \\ Spain. \\ * Colegio Universitario San Pablo C.E.U., \\ Montepríncipe, Boadilla del Monte, Madrid, Spain,
}

The orientation behaviour of three different interchanges in the grasshopper Eyprepocnemis plorans is cytologically analysed by means of $\mathrm{C}$-banding. The formation of chiasmata in interstitial regions produces metaphase $\mathrm{I}$ configurations different to those described in interchanges lacking such chiasmata, including frequent unbalanced orientations. Cytological evidence of undescribed orientations and segregations is presented. The structural effects of interstitial chiasmata and the validity of the rules governing the orientation of interchanges are discussed.

\section{INTRODUCTION}

Orientation behaviour of reciprocal translocation interchanges forming chiasmata in interstitial regions has not been as extensively studied as that of interchanges lacking them, so that most of the rules governing the orientation of multiples apply only to the latter type (Rickards, 1983b).

Chiasma localisation seems to be roughly maintained in the chromosomes involved in translocations. Thus the frequency of interstitial chiasmata depends on the material analysed. In particular, most cytological studies on interchange orientation deal with metaphase I structures showing terminal associations in the multiple and in the bivalents as well. This is the case in rye, cotton, Allium and cockroaches. In Drosophila and mice with a less strict localisation of chiasmata specific meiotic features do not allow an accurate cytogenetic study. Data on orientation behaviour of interstitial chiasmata forming interchanges therefore come mainly from segregation and fertility studies (Zimmering, 1955; de Boer, 1979; de Boer and van Beek, 1982). These studies have revealed that orientation behaviour of this type of translocations may be different from that of the best known ones.

In this paper we analyse cytologically the orientation behaviour of three different interchanges of Eyprepocnemis plorans forming chiasmata in interstitial regions, in an attempt to describe the characteristic types of co-orientating configurations and to test the validity of the rules proposed as governing the process.

\section{MATERIALS AND METHODS}

Three different reciprocal translocations have been studied by means of C-banding in the grasshopper Eyprepocnemis plorans. The A.1 interchange (fig. 1) involves one of the longest pairs of the complement ( $\mathrm{L}$ group) and the chromosome $\mathrm{M} 9$, the megameric pair in this species. It is a spontaneous translocation found in a male sample collected near Arenillas (Cádiz).

The other two, E.2411 (fig. 2) and E.1921 (fig. 3 ), were found in descendants of $X$-irradiated males. In E.2411 a long (L group) and a medium ( $\mathrm{M}$ group) chromosomes are involved. Both are unidentifiable in the normal complement but a quantitative difference in the centrometric C-bands of the $\mathrm{M}$ pair allowed us to individualise the two interstitial regions. E.1921 is a large interchange involving the longest chromosome of the complement (L1) and one medium ( $\mathrm{M}$ group) chromosome carrying a thin paracentric C-band in both members of the pair, which again allowed us to differentiate between the two interstitial regions.

For the 1921 translocation, several families of carrier individuals were obtained after three generations of backcrossing heterozygous males with standard homozygous females. Metaphase I configurations are studied in 18 males from these families. 

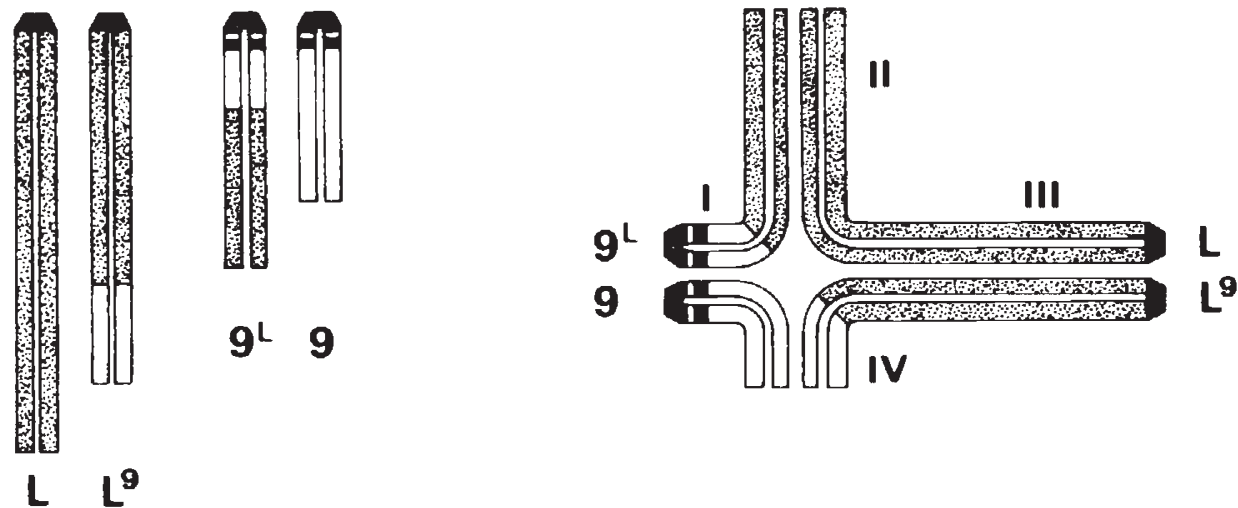

Figure 1 Diagram of the chromosomes involved in the interchange A.1 and pachytene cross showing the four cytologically distinguishable regions

Table 1 Metaphase I configurations of the A.1 translocation heterozygote

\begin{tabular}{|c|c|c|c|c|c|c|}
\hline I & II & $\begin{array}{l}\text { XTA } \\
\text { gion } \\
\text { III }\end{array}$ & IV & Configuration & $\begin{array}{l}\text { Fre- } \\
\text { quency }\end{array}$ & Co-orientation \\
\hline 0 & 1 & 1 & 1 & chain II & 27 & alternate/adjacent 1 \\
\hline 0 & 1 & 2 & 1 & chain II & 3 & \\
\hline 1 & 1 & 1 & 0 & chain I & 4 & \\
\hline 1 & 1 & 1 & 1 & ring & 5 & \\
\hline 1 & 1 & 2 & 1 & ring & 1 & \\
\hline 0 & 1 & 1 & 1 & chain II & 13 & adjacent 2 \\
\hline 0 & 1 & 1 & 1 & chain II & 13 & $3: 1 \mathrm{~L} 99 / \mathrm{L}$ \\
\hline 1 & 1 & 1 & 0 & chain I & 1 & \\
\hline 0 & 1 & 1 & 1 & chain II & 2 & $3: 1$ LL9/9 \\
\hline 0 & 1 & 1 & 0 & III + I & 29 & III alternate \\
\hline 0 & 1 & 2 & 1 & $I I I+I$ & 1 & \\
\hline 0 & 1 & 1 & 0 & $\mathrm{III}+1$ & 1 & III adjacent \\
\hline 0 & 1 & 1 & 1 & & 3 & indistinguishable \\
\hline 1 & 1 & 1 & 1 & & 1 & \\
\hline 1 & 1 & 1 & 1 & & 3 & \\
\hline
\end{tabular}

Testes were dissected in vivo and fixed in aceticacid:ethanol $1: 3$. The C-banding staining procedure was that described by Santos and Giráldez (1978).

\section{RESULTS}

Due to the telocentric nature of the chromosomes involved in the interchanges, chains and rings of four chromosomes-the most illuminating configurations for the study of orientation behaviour-depend on the formation of chiasmata in at least one of the interstitial regions. In this case, two types of chains can appear, open either at a translocated segment or at an interstitial one. These two types do not correspond exactly with those classified by John and Lewis (1965) in interchanges lacking chiasmata in interstitial regions as type I-when the extreme centromeres are nonhomologous-and type II when they are homologous. Nevertheless the chains in figures $4 a$, $4 \mathrm{c}-\mathrm{f}$ and $5 \mathrm{f}$ show homologous extreme centromeres so that we have called them type II and those 

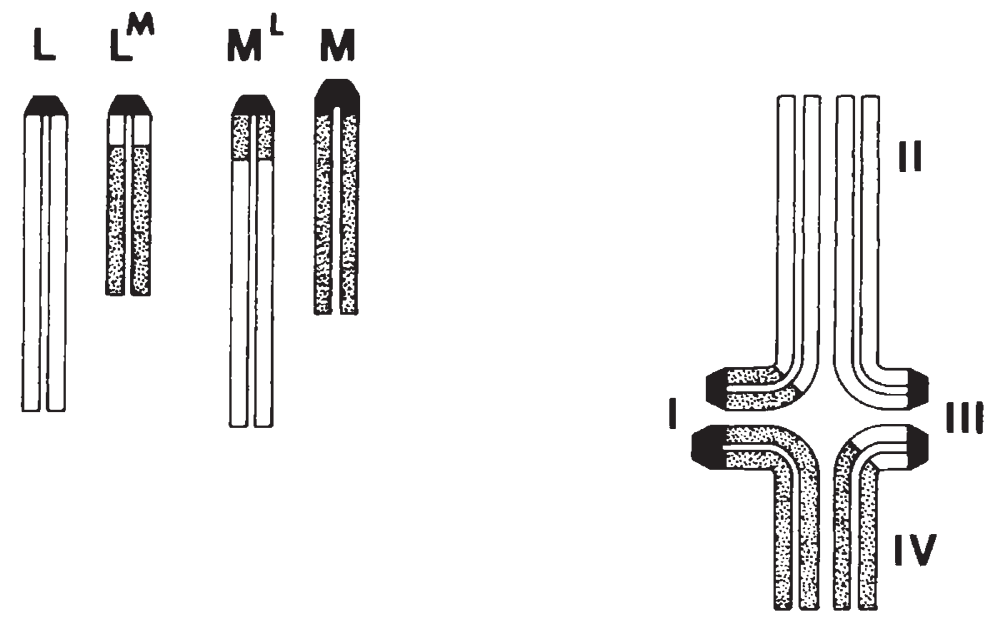

Figure 2 Diagram of the chromosomes involved in the translocation 2411 and pachytene cross showing the four cytologically distinguishable regions.

Table 2 Metaphase I configurations of the E.2411 translocation heterozygote

\begin{tabular}{|c|c|c|c|c|c|c|}
\hline I & II & $\begin{array}{l}\text { XTA } \\
\text { gion }\end{array}$ & IV & Configuration & $\begin{array}{l}\text { Fre- } \\
\text { quency }\end{array}$ & Co-orientation \\
\hline 0 & 1 & 1 & 1 & chain II & 6 & alternate/adjacent 1 \\
\hline 1 & 1 & 0 & 1 & chain II & 6 & \\
\hline 1 & 1 & 1 & 0 & chain I & 2 & \\
\hline 1 & 1 & 1 & 1 & ring & 3 & \\
\hline 0 & 1 & 1 & 1 & chain II & 5 & adjacent 2 \\
\hline 1 & 1 & 0 & 1 & chain II & 4 & \\
\hline 1 & 1 & 1 & 1 & ring & 1 & \\
\hline 1 & 1 & 0 & 1 & chain II & 1 & $3: 1$ \\
\hline 0 & 1 & 0 & 1 & $2^{\mathrm{II}}$ & 80 & \\
\hline 0 & 1 & 1 & 1 & chain II & 7 & linear \\
\hline 1 & 1 & 0 & 1 & chain II & 4 & \\
\hline 0 & 1 & 1 & 1 & & 1 & indistinguishable \\
\hline 1 & 1 & 0 & 1 & & 2 & \\
\hline 1 & 1 & 1 & 1 & & 1 & \\
\hline
\end{tabular}

lacking chiasmata in a translocated segment were denominated as type $\mathrm{I}$.

When chiasmata in interstitial regions form, the alternate and adjacent 1 orientations become indistinguishable (McClintock, 1945; Burnham, 1956). There is genetical evidence of the actual occurrence of both (Zimmering, 1955; Burnham, 1956, 1962; Searle et al., 1971; de Vries, 1983) since the respective segregations of markers that they produce are different. Cytologically it is also possible to distinguish between the two types if one is able to identify the four chromosomes in the multi- ple. This is the case with the type II chains of one of the 1921 carrier males in which the translocated L1 member possessed a thick centromeric C-block whilst its homologue (non-translocated L1) showed a thinner band (figs $4 \mathrm{c}$ and $4 \mathrm{e}$ ). Consequently, the chain in figure $4 \mathrm{e}$ corresponds to adjacent 1 orientation since the translocated L1 and the non-translocated $\mathrm{M}$ chromosomes orient to the same pole. However, due to the indeterminacy introduced by the $\mathrm{M}$ centromeres, in type I chains and rings of the same individual it is not possible to differentiate alternate from adjacent 1 . 

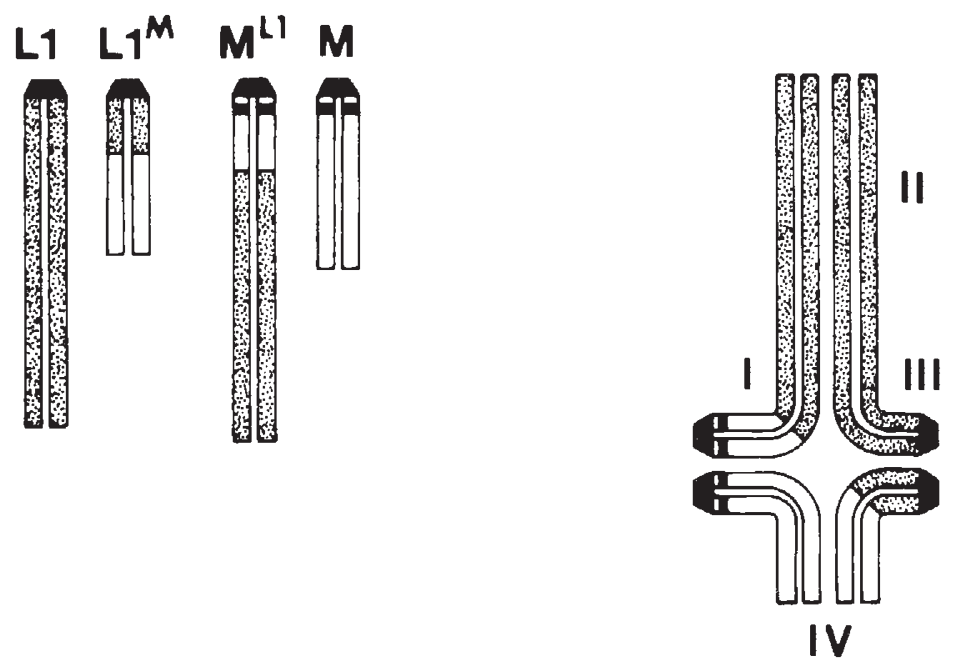

Figure 3 Diagram of the chromosomes involved in the interchange 1921 and pachytene cross showing the four cytologically distinguishable regions.

Table 3 Metaphase I configurations of the E.1921 translocation heterozygote

\begin{tabular}{|c|c|c|c|c|c|c|}
\hline I & II & $\begin{array}{l}\text { XTA } \\
\text { gion }\end{array}$ & IV & Configuration & $\begin{array}{l}\text { Fre- } \\
\text { quency }\end{array}$ & Co-orientation \\
\hline 0 & 1 & 1 & 1 & chain II & 18 & alternate/adjacent 1 \\
\hline 0 & 2 & 1 & 1 & chain II & 4 & \\
\hline 1 & 1 & 0 & 1 & chain II & 9 & \\
\hline 1 & 1 & 1 & 0 & chain I & 9 & \\
\hline 1 & 2 & 1 & 0 & chain I & 3 & \\
\hline 1 & 1 & 1 & 1 & ring & 52 & \\
\hline 1 & 2 & 1 & 1 & ring & 11 & \\
\hline 0 & 1 & 1 & 1 & chain II & 8 & adjacent 2 \\
\hline 0 & 2 & 1 & 1 & chain II & 4 & \\
\hline 1 & 1 & 0 & 1 & chain II & 1 & \\
\hline 1 & 1 & 1 & 1 & ring & 3 & \\
\hline 1 & 2 & 1 & 1 & ring & 1 & \\
\hline 0 & 1 & 1 & 1 & chain II & 16 & $3: 1$ \\
\hline 0 & 2 & 1 & 1 & chain II & 4 & \\
\hline 1 & 1 & 0 & 1 & chain II & 5 & \\
\hline 1 & 2 & 0 & 1 & chain II & 2 & \\
\hline 0 & 1 & 0 & 1 & $2^{\mathrm{II}}$ & 34 & \\
\hline 0 & 2 & 0 & 1 & $2^{I I}$ & 6 & \\
\hline 0 & 1 & 1 & 1 & & 3 & indistinguishable \\
\hline 0 & 2 & 1 & 1 & & 1 & \\
\hline 1 & 1 & 0 & 1 & & 2 & \\
\hline
\end{tabular}

In all the three interchanges, type I chains orientate almost invariably in an alternate/adjacent 1 manner (fig. 4b). However both type II chains and rings display greater variation giving also $3: 1$ (figs $4 \mathrm{~d}$ and $5 \mathrm{a}$ ) and adjacent 2 (figs $4 \mathrm{c}$, $4 \mathrm{f}$ and $5 \mathrm{~b}$ ) orientations (see tables $1,2,3$ and 4 ).
$3: 1$ unbalanced orientations in type II chains have been cytologically observed in grasshopper interchanges structurally similar to these described here (Carothers, 1931; Sarkar, 1955; Wise and Rickards, 1977) but they never have been reported in rings (fig. 5a). In our material, 3:1 rings appear 

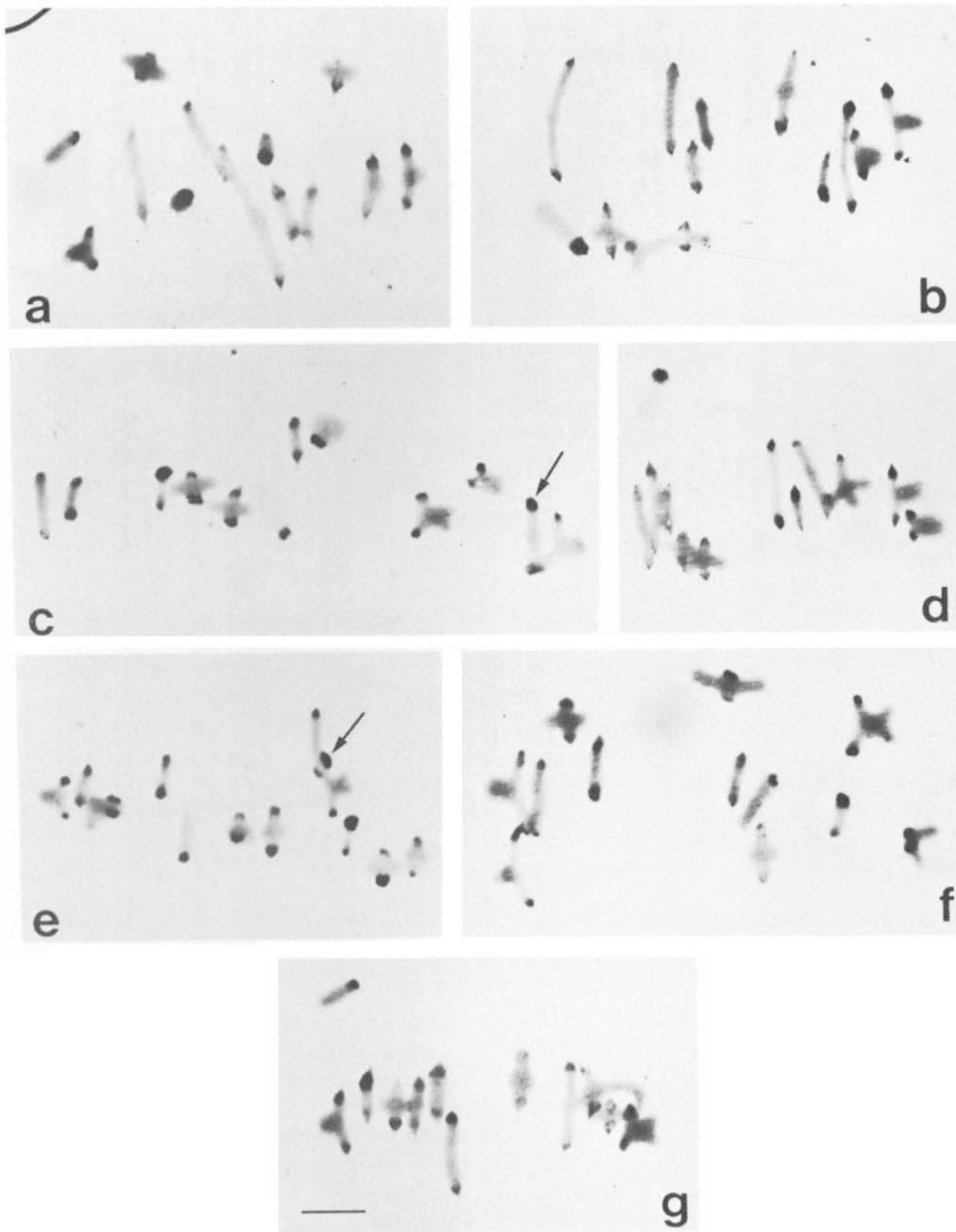

Figure 4 Metaphase I cells of the 1921 interchange showing different quadrivalent co-orientations. Note that there is a considerable quantitative variation in the centromeric heterochromatin of several chromosome pairs. (a) Alternate/adjacent 1 type $I I$ chain. (b) Alternate/adjacent 1 type I chain. (c) Adjacent 2 type II chain showing an enlarged block of centromeric C-heterochromatin in the L1 translocated chromosome (arrowed). (d) 3:1 type II chain. (e) Alternate/adjacent 1 type II chain showing a thick centrometic C-band in the translocated Ll (arrowed). (f) Adjacent 2 type Il chain. (g) Alternate/adjacent 1 ring. Bar $=10 \mu \mathrm{m}$.

repeatedly in the 1921 heterozygotes and evidence of $3: 1$ segregation of such rings has been found at anaphase I and metaphase II (fig. 5c).

Adjacent 2 orientations have been observed in chains of the A.1 and E.2411 translocations and in rings and chains of the 1921 interchange (figs $4 c, 4 f, 5 b$ and $5 d$ ). It is generally accepted that this type of orientation is precluded by the presence of chiasmata in interstitial regions, although there are some exceptions (for review, see Rickards, 
Table 4 Metaphase I configurations of 18 heterozygotes carrying the 1921 interchange. Pooled data

\begin{tabular}{|c|c|c|c|c|c|c|}
\hline l & 11 & $\begin{array}{l}\text { XTA. } \\
\text { gion }\end{array}$ & IV & Configuration & $\begin{array}{l}\text { Fre- } \\
\text { quency }\end{array}$ & Co-orientation \\
\hline 1 & 1 & 1 & 1 & ring & 958 & alternate/adjacent 1 \\
\hline 1 & 2 & 1 & 1 & ring & 452 & \\
\hline 0 & 1 & 1 & 1 & chain II & 133 & \\
\hline 0 & 2 & 1 & 1 & chain II & 82 & \\
\hline 1 & 1 & 0 & 1 & chain II & 63 & \\
\hline 1 & 2 & 0 & 1 & chain II & 33 & \\
\hline 1 & 1 & 1 & 0 & chain I & 83 & \\
\hline 1 & 2 & 1 & 0 & chain I & 8 & \\
\hline 0 & 1 & 1 & 0 & $\mathrm{III}+\mathrm{I}$ & 2 & III alternate \\
\hline 0 & 2 & 1 & 0 & $111+1$ & 3 & \\
\hline 1 & 1 & 1 & 1 & ring & 50 & adjacent 2 \\
\hline 1 & 2 & 1 & 1 & ring & 83 & \\
\hline 0 & 1 & 1 & 1 & chain II & 85 & \\
\hline 0 & 2 & 1 & 1 & chain II & 84 & \\
\hline 1 & 1 & 0 & 1 & chain II & 36 & \\
\hline 1 & 2 & 0 & 1 & chain II & 51 & \\
\hline 1 & 1 & 1 & 1 & ring & 100 & $3: 1$ \\
\hline 1 & 2 & 1 & 1 & ring & 76 & \\
\hline 0 & 1 & 1 & 1 & chain II & & \\
\hline 0 & 2 & 1 & 1 & chain II & 54 & \\
\hline 1 & 1 & 0 & 1 & chain II & 40 & \\
\hline 1 & 2 & 0 & 1 & chain II & 22 & \\
\hline 1 & 1 & 1 & 0 & chain I & 1 & \\
\hline 0 & 1 & 1 & 1 & chain II & 38 & linear \\
\hline 0 & 2 & 1 & 1 & chain II & 33 & \\
\hline 1 & 1 & 0 & 1 & chain II & 16 & \\
\hline 1 & 2 & 0 & 1 & chain II & 20 & \\
\hline 0 & 1 & 0 & 1 & $2^{I I}$ & 184 & \\
\hline 0 & 2 & 0 & 1 & $2^{I I}$ & 176 & \\
\hline
\end{tabular}

1983b). Here we present cytological evidence showing that adjacent 2 orientation and segregation can occur in the presence of interstitial chiasmata. Although dealing with regions of comparable length, the behaviour of the three interchanges appears to differ. While an interstitial chiasma in region I of A.1 invariably determines the co-orientation of the $\mathrm{M}$ centromeres (table 1), in the interchanges E.2411 and 1921 homologous centromeres closely linked by interstitial chiasmata in regions I and/or III can orientate either to the same or to the opposite poles (tables 2, 3 and 4). This variable behaviour also appears when the interstitial chiasma in region I of the 1921 interchange is extremely proximal, lying between the $\mathrm{M}$ centromeres and the pericentric C-band (fig. $5 \mathrm{f}$ ).

Among the metaphase I cells-carefully selected to be at a late stage-some undetermined orientations were found in which at least one of the centromeres in the multiple was not clearly facing any pole (fig. $5 \mathrm{f}$ ). Although it is difficult to establish a causal relationship, these figures could correspond to the amphitelic divisions found in later meiotic stages as telophase I involving chromosomes similar in size to the short (translocated $\mathrm{L} 1$, non-translocated $\mathrm{M}$ ) and the long (nontranslocated L1, translocated $\mathrm{M}$ ) members of the interchange (fig. 5e). Amphitelic divisions cannot be totally attributed to univalence at metaphase I since the frequency of univalents in this phase is negligible, null for long chromosomes, whereas chromatid segregation at telophase is more frequent.

Another type of metaphase I configurations found in the translocations of E.plorans is the "linear" orientation in type II chains of the E.2411 and 1921 interchanges (fig. $5 \mathrm{~g}$ ). These undetermined configurations have been reported in several materials, being most frequent in interchanges with chiasmata in interstitial regions. The linear orienta- 


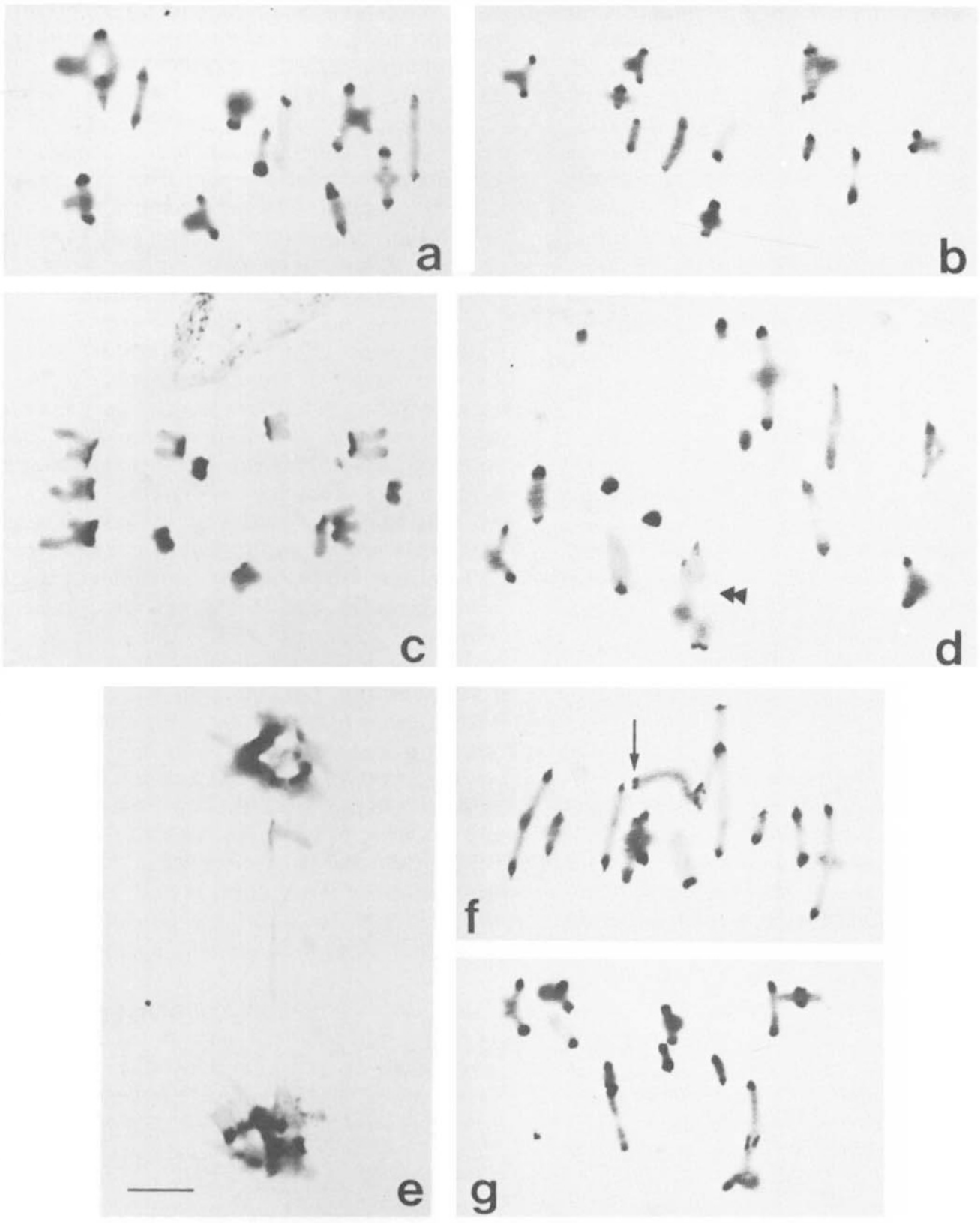

Figure 5 Orientations and segregations of the 1921 interchange. (a) Metaphase I showing a 3:1 ring. (b) Metaphase I showing an adjacent 2 ring. (c) Metaphase II segregation from a 3:1 ring. Note the three chromosomes with unequal chromatids. (d) Early anaphase I showing an adjacent 2 ring segregation (double arrowhead). (e) Telophase II showing an equationally dividing long chromosome. (f) Undetermined metaphase I orientation of a type II chain. Note the location of the interstitial chiasma between the co-orientating $M$ centromeres and the pericentric C-band. (g) Linear type II chain. Bar $=10 \mu \mathrm{m}$.

tions found in a translocation of the grasshopper Melanoplus by Wise and Rickards (1977) are considered by these authors as alternate/adjacent 1 in which the interstitial chiasma is so close to the centromeres that it makes it difficult to distinguish their mode of orientation. However, the use of C-banding in E.plorans, allowing a more reliable identification of the centromeric regions, rather suggests that the centromeres in the middle of the chain do not co-orient at all. 
In order to ascertain the mode of segregation of linear chains, a parallel study of metaphase I and metaphase II was carried out in one 1921 translocation heterozygote. The high degree of chromosome contraction at metaphase II does not allow us to identify the four chromosomes involved in the rearrangement thus only the total number of chromosomes and the number of chromosomes with unequal chromatids, evidence of interstitial chiasmata, per cell were scored. The results indicate that adjacent 2 segregation of linear chains is unlikely (table 5). The number of cells available is not enough to distinguish between alternate/adjacent 1 and 3:1 segregations since the data fit in both cases.

It should be pointed out that there is continuous variation in the angle determined by an hypothetical straight line linking the two centromeric ends in a type II chain and the polar axis. Within this range, the linear chains represent the extreme case of total alignment (see figs $4 \mathrm{a}, 4 \mathrm{e}$ and $5 \mathrm{~g}$ for comparison).

\section{DISCUSSION}

The idea that the orientation of quadrivalents is based on co-orientation of pairs of centromeres is generally accepted although there has been lately a considerable controversy about the subject since Boussy (1982) proposed a tridimensional interpretation for alternate rings, implying the co-orientation of a given centromere with its two adjacent ones.
Following this rule, if one considers that it is only possible to establish tension forces between centromeres axially aligned (Cochran, 1983; Endrizzi et al., 1983; Rickards, 1983a), in some of the configurations of E.plorans interchanges shown here it is difficult to decide which are the pairs of co-orientating centromeres. This is the case with alternate/adjacent 1 type II chains (see fig. $4 a$ ) in which there are several possibilities: (a) each centromere co-orientates with its homologue, hence there should be an axial force between the inner centromeres and an oblique force between the extreme ones. (b) oblique forces are established between each extreme centromere and one of the co-orientating inner pair. In this case it is necessary to propose that each inner centromere is simultaneously co-orientating with its homologue and with one non-homologous extreme centromere. (c) all tension forces-axial or otherwise-originate star-like from an application point that coincides in location with the interstitial chiasma linking the inner centromeres. This point was proposed by Wise and Rickards (1977) and seems to be the most adequate to explain the intermediate configurations (fig. 4e) in which neither the extreme centromeres nor the inner ones appear axially oriented.

In this type of interchanges therefore it is necessary to propose either the existence of oblique forces acting in the orientation of that they do not follow the rule of co-orientation by pairs of centromeres. The latter point is best illustrated when the $3: 1$ type II chains are considered (fig. 4d). Here it is clear that the single centromere is co-

Table 5 Comparison between the observed metaphase II and expected metaphase 1 segregations in one 1921 translocation heterozygote in order to ascertain the mode of segregation of the linear chains

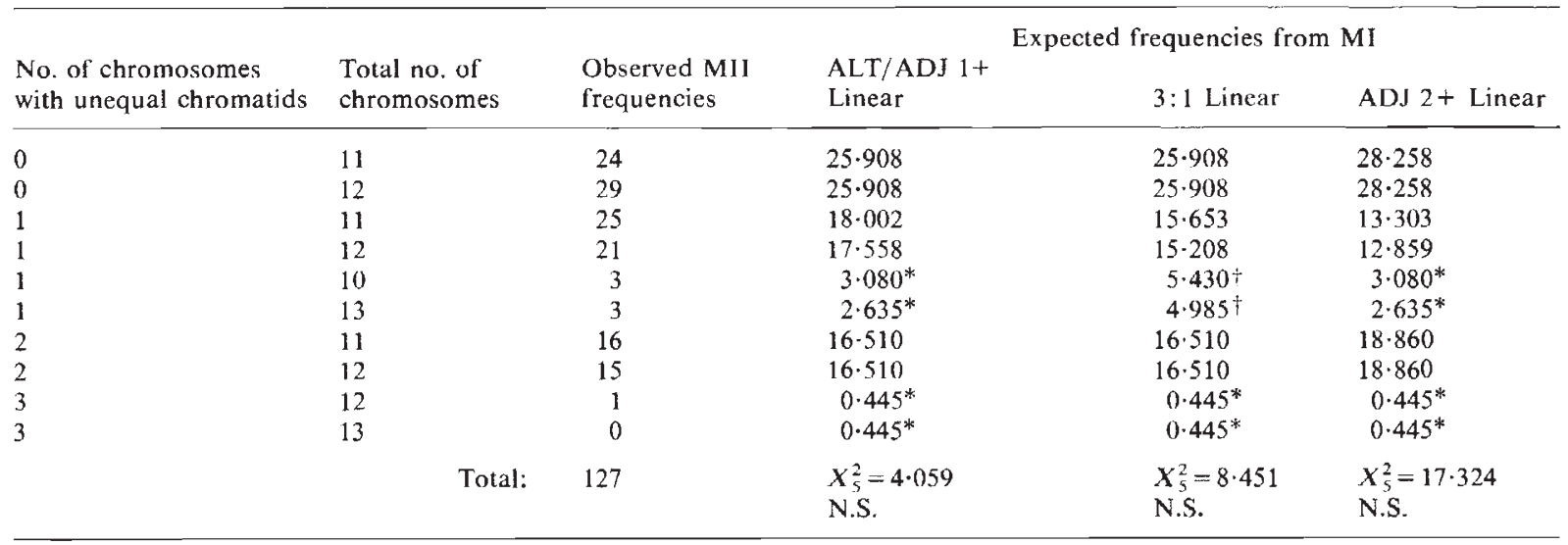

* Classes $<5$ excluded.

$\dagger$ Excluded classes for adequate comparison with the other two columns. If included in the test: $X_{7}^{2}=10 \cdot 329$ N.S. 
orientating with the remaining three. Again, only the tension force established between the central homologous centromeres is axial whereas those between non-homologous one should be oblique. It is not possible to consider, as Endrizzi et al. (1983) propose, that each pair of co-orientating centromeres is aligned along a single chromosome spindle fibre axis.

It is noteworthy that in interchanges lacking interstitial chiasmata, both the adjacent 1 in type II chains and the $3: 1$ unbalanced orientations are considered unstable, although both configurations are stable in the presence of interstitial chiasmata (see fig. 6 for comparison). Assuming that a multiple is wholly stable if each centromere has at least one other adjacent linked centromere oriented to the opposite pole (Rickards, 1977), the effect of interstitial chiasmata could be simply to supply new ways of direct linkage between centromeres. In other words, they make adjacent centromeres that would not be so in the absence of such chiasmata. Indeed, a single interstitial chiasma directly interconnects all the centromeres in a multiple of four, allowing chains to orient as adjacent 1 (strictly alternate/adjacent 1) and 3:1 (fig. 6).

The considerable frequency of adjacent 2 chains and/or rings found in the $E$. plorans interchanges reveals that suppression of the nondisjunction of homologous centromeres by interstitial chiasmata is not a general rule. Nevertheless, there are marked differences in the behaviour of the three translocations. In the A.1 interchange the presence of one chiasma in the short interstitial region I actually precludes adjacent 2 orientation although chains with a single chiasma in the long interstitial region III can orientate in this way (table 1). Such behaviour can be explained under the hypothesis of Arana et al. $(1980,1982)$ - the shorter the distance between two centromeres, the higher their probability of co-orientation-if the physical distance between the M9 centromeres is short enough to assure their co-orientation. Using the terminology of Vosselman (1981), the physical distance between the M9 centromeres is a "coorientation determining distance". Thus, a single chiasma in the long interstitial region III can determine a much longer physical distance between the L centromeres allowing them to migrate, in some cases, to the same pole. Similar results would be expected if we assume that a proximal chiasma in an interstitial region is more effective in maintenance of the "back to back" disposition of the homologous centromeres than a chiasma located closer to the break-point and thus more effective in the pre-orientation processes (Rickards, 1983b).

On another hand, based on metaphase II segregation combined with fertility studies, de Boer (1979) proposed that an interstitial chiasma extremely proximal to the homologous centromeres precluded their migration to opposite poles, causing them to behave as an unit. Our data do not support such a hypothesis since the M9 centromeres in the A.1 interchange always coorientate in the presence of one chiasma in the interstitial region I. Such a chiasma is unavoidably proximal due to the short length of this region.
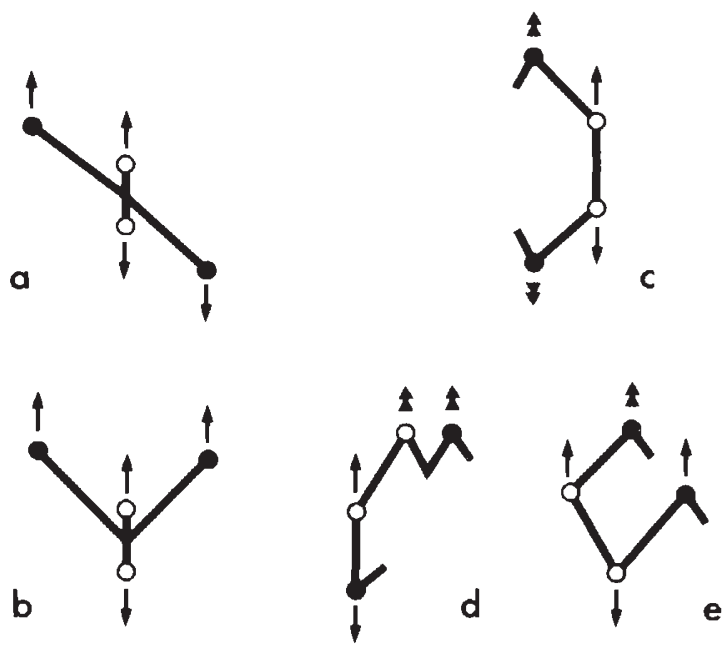

Figure 6 Comparison of the adjacent $1(\mathrm{a}, \mathrm{c})$ nd $3: 1(\mathrm{~b}, \mathrm{~d}, \mathrm{e})$ quadrivalent co-orientations between type II chains with (a, b) and without (c, d, e) interstitial chiasma. (c), (d) and (e) from Rickards (1983b). Arrows mark stable centromeres and double arrowheads unstable centromeres. 
Similarly in rings and chains of interchanges 2411 and 1921 homologous centromeres closely linked by interstitial chiasmata in regions I and/or III can orientate either to the same or to the opposite poles (figs $4 \mathrm{~g}, 5 \mathrm{a}, 5 \mathrm{~b}$ ). Even in the most extreme case of proximal location of the interstitial chiasma-when it lies between the $\mathrm{M}$ centromeres and the pericentric C-band in the region I of the 1921 interchange-the homologous M centromeres can co-orientate (fig. 5f).

Unlike the A.1 translocation, the 2411 and 1921 interchanges show a considerable frequency of adjacent 2 in the presence of chiasmata in the short interstitial regions (tables 2, 3 and 4). It is evident that the "co-orientation determining distance" varies amongst interchanges or perhaps may never be achieved. In these cases, the distance hypothesis cannot be easily applied. It is possible that in the 1921 and 2411 translocations the distance between non-homologous centromeres is closer to the distance between homologous ones than in other grasshopper interchanges including the A.1. Thus if the relative intercentromeric physical distances have an influence in orientation, as in the Arana et al. (1980, 1982) hypothesis, this should lead to a higher probability of non-homologous co-orientation. Nevertheless this explanation alone seems inadequate to account for the marked differences in behaviour observed. Other factors may be involved in the process of orientation and these are the subject of further investigations using these translocations.

Acknowledgements This work has been partially supported by the Comisión Asesora de Investigación Científica y Técnica.

\section{REFERENCES}

ARANA, P., SANTOS, J. L. ANI GIRALDEZ, R. 1980. Chiasma interference and centromere co-orientation in a spontaneous translocation heterozygote of Euchorthippus pulvinatus gallicus (Acrididae; Orthoptera). Chromosoma, 78, $327-340$.

ARANA, P., SANTOS, J. L., HENRIQUES-GII, N. AND GIRALDEZ, R. 1982. Centromere co-orientation in a spontancous translocation heterozygote of Euchorthippus pulvinatus gallicus (Acrididae; Orthoptera). Genetica, 58, 81-84.
DE BOER, P. 1979. Proximal chiasma location within an interstitial chromosome segment, a likely correlate of adjacent-2 segregation causing multivalents in the mouse. Environmental Health Perspectives, 31, 137-140.

DE BOER, P. AND VAN BEEK, M. E. A. B. 1982. Meiosis in T70H translocation trisomic male mice. I. Meiotic configurations and segregation. Chromosoma, 87, 303-312.

BousSY, I. A. 1982. "Alternate-2" disjunction does not exist. Genetics, 100, 505-509.

BURNHAM, C. R. 1956. Chromosomal interchanges in plants. Bot. Rev., 22, 419-552.

BURNHAM, C. R. 1962. Discussions in Cytogenetics, Burgess Publ. Co, Minneapolis, 375 pp.

CAROThERS, E. 1931. The maturation and segregation of heteromorphic homologous chromosomes in Acrididae (Orthoptera). Biol. Bull., 61, 324-349.

COCHRAN, D. G. 1983. Alternate-2 disjunction in the German cockroach. Genetics, 104, 215-217.

ENDRIZZI, I. F., RAY, D. T. AND GATHMAN, A. C. 1983. Centromere orientation of quadrivalents of heterozygous translocations and an autoploid of Gossypium hirsutum L., Genetics, 105, 723-731.

IOHN, B. AND LEWIS, K. R. 1965. The meiotic System. Protoplasmatologia, Vol. 6, FL, Springer, New York, 335 pp.

MCCLINTOCK, B. 1945. Neurospora. I. Preliminary observations of the chromosomes of Neurospora crassa. Amer. J. Bot., 32, 671-678.

RICKARDS, G. K. 1977. Prometaphase I and anaphase $I$ in an interchange heterozygote of Allium triquetrum (Liliaceae). Chromosoma, 64, 1-23.

RICKARDS, (i. K. 1983a. Alternate-1 and alternate- 2 orientations in interchange (reciprocal translocation) quadrivalents. Genetics, 104, 211-213.

RICKARDS, G. K. $1983 b$. Orientation behavior of chromosome multiples of interchange (reciprocal translocation) heterozygotes. Ann. Rev. Genet., 17, 443-498.

SANTOS, J. L. AND GIRALDEZ, R. 1978. The elfect of C-heterochromatin in chiasma terminalisation in Chorthippus biguttulus L. (Acrididae, Orthoptera). Chromosoma, 70, 59-66.

SARKAR, 1. 1955. A translocation heterozygote in the grasshopper Gesonula punctifrons. J. Hered., 46, 157-160.

SEARLE, A. G., FORD, C. E. AND BEECHEY, C. V. 1971. Meiotic disjunction in mouse translocations and the determination of the centromere position. Gen. Res., 18, 215-235.

voSSELMAN, L. 1981. Meiotic segregation of five different reciprocal translocations in the onion fly Hylemya antiqua (Meigen). Chromosoma, 81, 727-738.

DE VRIES, J. N. 1983. High recombination between the breakpoint of a reciprocal translocation in rye (Secale cereale L.) and an interstitially located gene. Theor. Appl. Genet., 66, 329-339.

WISE, D. AND RICKARDS, G. K. 1977. A quadrivalent studied in living and fixed grasshopper spermatocytes. Chromosoma, 63, 305-315.

ZIMMIRING, S. 1955. A genetic study of segregation in a translocation heterozygote in Drosophila. Genetics, 40, 809825 . 